\title{
Event-by-event Particle Yield Ratio Fluctuations in the CBM and NA49 Experiments
}

\author{
Dmytro Kresan* for the CBM and NA49 collaborations \\ GSI, Darmstadt \\ E-mail: D.Kresanegsi.de
}

\begin{abstract}
Non-statistical event-by-event fluctuations are considered an important signal for the critical endpoint of the QCD phase diagram. Event-by-event fluctuations of different observables are thus investigated in detail in current experiments. In this contribution, a study of the centrality dependence of event-by-event fluctuations of particle yield ratios by the NA49 experiment in $\mathrm{Pb}+\mathrm{Pb}$ collisions at $158 \mathrm{~A} \mathrm{GeV}$ beam energy is presented for the first time. An increase of the absolute values of dynamical fluctuations towards lower centralities is observed. The influence of resonance decays will be discussed.

Event-by-event fluctuations are an important observable to be studied at the future CBM experiment at FAIR. CBM will investigate the intermediate region of the QCD phase diagram in great detail searching for the first order phase transition line and the expected critical endpoint. It is therefore important to investigate the sensitivity of the CBM detector to particle ratio fluctuations in $\mathrm{Au}+\mathrm{Au}$ collisions at 10-45 AGeV beam energy. Detailed simulation studies will be presented.
\end{abstract}

5th International Workshop on Critical Point and Onset of Deconfinement - CPOD 2009,

June 08 - 122009

Brookhaven National Laboratory, Long Island, New York, USA

\footnotetext{
${ }^{*}$ Speaker.
} 


\section{Introduction}

Recent lattice QCD calculations expect a first order phase transition from hadronic to partonic degrees of freedom at finite temperature and baryon chemical potential [1,2]. This first order transition line ends with a critical endpoint [3]. The search for either the first order phase transition or the critical endpoint is a challenging task in modern high energy heavy-ion physics.

Due to density fluctuations at the critical point or in the coexistance region at a first order phase transition, fluctuations in particle yields and kinematic properties may occur. Indeed, in lattice QCD calculations the quark number susceptibility develops a peak at the critical temperature when increasing the quark chemical potential and thus approaching the expected critical point (figure 1) [4]. Fluctuations as a measure of susceptibilities [5] should than be enhanced.



Figure 1: The quark number susceptibility as a function of temperature for different quark chemical potentials [4].

In this work we will concentrate on event-by-event fluctuations of particle yield ratios, such as kaon to pion $(\mathrm{K} / \pi)$, proton to pion $(\mathrm{p} / \pi)$ and kaon to proton $(\mathrm{K} / \mathrm{p})$ ratios. Recently, the NA49 and STAR collaborations have published their results on the energy dependence of the dynamical fluctuations of $\mathrm{K} / \pi$ and $\mathrm{p} / \pi$ ratios [6,7]. The measured dependence is illustrated in figure 2 .

The dynamical fluctuations of the $\mathrm{K} / \pi$ ratio increase towards lower collision energies and are not reproduced by the UrQMD model, while the $\mathrm{p} / \pi$ ratio fluctuations from the data analysis are in good agreement with model calculations.

In [6] it was discussed, that this increase of the $\mathrm{K} / \pi$ ratio fluctuations towards lower beam energies could be associated with a decrease of particle multiplicities. Thus one would like to study the centrality dependence of the fluctuation signal in order to understand the behaviour of this observable with the changing average multiplicity of the accepted tracks. In addition, it is neccessary to remeasure the low energy region with better experimental resolution and larger acceptance to provide high quality data on this observable, which is one of the goals of the future CBM experiment. 


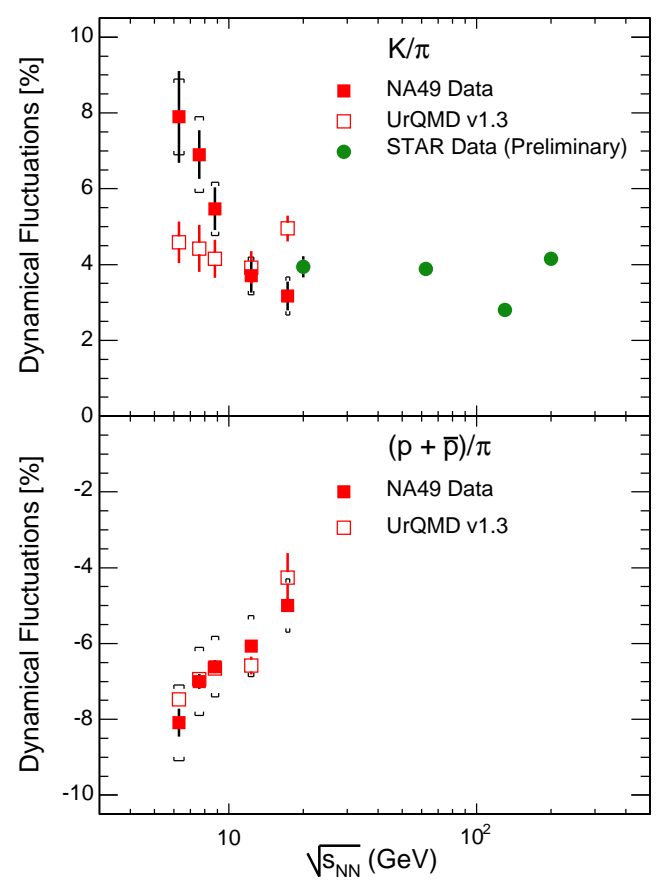

Figure 2: Energy dependence of the dynamical fluctuations of the $K / \pi$ and $p / \pi$ ratios in central $A+A$ collisions published by the NA49 and STAR experiments $[6,7]$.

\section{Centrality Dependence of Particle Ratio Fluctuations in NA49}

NA49 is a fixed target experiment at the CERN SPS. Measurements of $p+p, p+A$ and A + A collisions were recordered at different energies. NA49 is a large acceptance hadron spectrometer which includes two dipole magnets for momentum determination, two vertex TPCs for the measurement of vertices and particle identification (PID) using $d E / d x$, two main TPCs for PID with $d E / d x$, two TOF walls for PID at midrapidity using time-of-flight and a Zero Degree Calorimeter for centrality determination. A detailed description of the NA49 detector can be found in [8].

In the current analysis, the specific energy loss $d E / d x$, measured by the main TPCs of NA49 was used for particle identification. The NA49 spectrometer works in the relativistic rise region of the Bethe Bloch parametrisation of $d E / d x$ [9]. This implies some restrictions on the PID performance, namely a lower momentum cut of $3 \mathrm{GeV} / \mathrm{c}$ and no possibility of PID on the track-by-track level. Thus, particles have to be identified on a statistical basis by extracting the relative yields in a single event using the Maximum Likelihood Method. The data analysis technique is described in detail in $[10,11]$.

In this section we will focus on the measured centrality dependence of the dynamical fluctuations of particle yield ratios. The centrality of an A + A collision was determined by measuring the total energy of the projectile spectators deposited in the Zero Degree Calorimeter of NA49. The dynamical fluctuations of a particle yield ratio are defined as the geometrical difference between the relative width ( $\sigma=\mathrm{RMS} / \mathrm{MEAN})$ of the eventwise ratio distribution for data $\left(\sigma_{\text {data }}\right)$ and mixed $\left(\sigma_{m i x}\right)$ events: 


$$
\sigma_{d y n}=\operatorname{sign}\left(\sigma_{d a t a}-\sigma_{m i x}\right) \sqrt{\left|\sigma_{d a t a}^{2}-\sigma_{m i x}^{2}\right|}
$$

\subsection{Centrality Bin Size}

The main motivation to use particle yield ratios in the fluctuation analysis is that in first approximation volume fluctuations are cancelled in the ratio. Nevertheless, the fluctuations of the projectile and target participants in $\mathrm{Pb}+\mathrm{Pb}$ collisions may have an influence on the measured signal, thus more detailed studies had been performed on the subject of the dependence of dynamical fluctuations on the selected centrality bin size. In the published NA49 results on the energy dependence of the $3.5 \%$ most central $\mathrm{Pb}+\mathrm{Pb}$ collisions were used in the analysis $[6,10]$.

The distribution of the total energy of the projectile spectators deposited in the Zero Degree Calorimeter of NA49 is shown in figure 3 (left plot). The centrality was integrated in the following bins: $(0-3) \%,(0-3.5) \%,(0-5) \%, \ldots,(0-20) \%$ most central $\mathrm{Pb}+\mathrm{Pb}$ collisions at $158 \mathrm{~A} \mathrm{GeV}$ beam energy. The observed dependence of the $\mathrm{K} / \pi$ ratio fluctuations is shown in figure 3 (right plot).
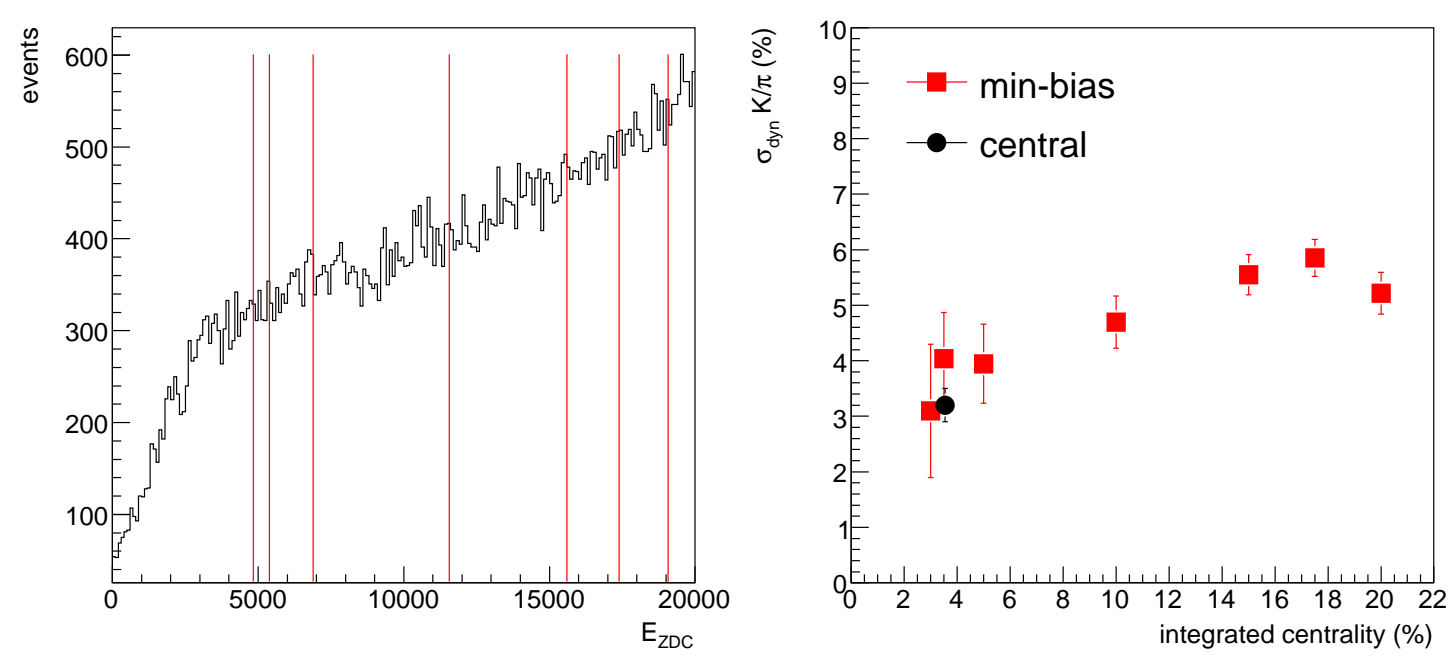

Figure 3: Left picture: distribution of the energy deposited in the Zero Degree Calorimeter (ZDC) of NA49 in $\mathrm{Pb}+\mathrm{Pb}$ collisions at $158 \mathrm{~A} \mathrm{GeV}$ beam energy. Vertical lines show cuts, which correspond to $3 \%, 3.5 \%$, $5 \%, 10 \%, 15 \%, 17.5 \%$ and $20 \%$ centrality bin size. Right picture: dynamical fluctuations of the $\mathrm{K} / \pi$ ratio as a function of the centrality bin size in $\mathrm{Pb}+\mathrm{Pb}$ collisions at $158 \mathrm{~A} \mathrm{GeV}$ beam energy.

The results are in good agreement with the top SPS beam energy point from [6]. As has been discussed before, the fluctuations of the number of projectile and target participants have an effect on the measured signal, and a slight increase of about $2 \%$ of fluctuations with increasing integrated centrality of $\mathrm{Pb}+\mathrm{Pb}$ collisions is observed as a general trend. However, the difference between the values of the dynamical fluctuations of the kaon to pion yield ratio in case of using $3.5 \%$ and $5 \%$ most central $\mathrm{Pb}+\mathrm{Pb}$ collisions is small. So, in order to gain statistics, which is important for the errors, the centrality dependence of the dynamical fluctuations can be studied using a centrality bin size of $5 \%$. 


\subsection{Simulations with UrQMD}

Particle identification on the statistical basis can introduce a bias to the event-by-event fluctuations. This bias might be larger in semi-peripheral $\mathrm{Pb}+\mathrm{Pb}$ collisions, since in this case the average track multiplicity decreases and the identification procedure becomes more difficult. It becomes particularly challenging if single particle multiplicities in the acceptance approach zero. As no negative numbers can be allowed for stable fit results, the eventwise $\mathrm{K} / \pi$ distribution develops a spike at zero $[6,11]$.

In order to find out the working limits for the analysis of the centrality dependence of $\mathrm{Pb}+\mathrm{Pb}$ collisions, simulations with the UrQMD model [12] have been performed. The centrality of a collision was determined using the provided value of the impact parameter. Particles, generated by the model, were processed through the acceptance filter of NA49, which incorporates the specific track cuts used in the current data analysis. For each accepted track, a $d E / d x$ value was simulated using a parametrization of the measured $d E / d x$ distribution in NA49. The accepted tracks with their simulated $d E / d x$ response were processed through the same analysis routines as the tracks from data, namely the event-by-event fit of the eventwise $d E / d x$ distribution (E-b-e fit). As an unbiased reference, particle identification based on the particle type provided by the UrQMD model was used, so called Monte Carlo identification (MC PID). The extracted centrality dependence of the dynamical fluctuations of the kaon to pion yield ratio for these two cases is shown in figure 4 .

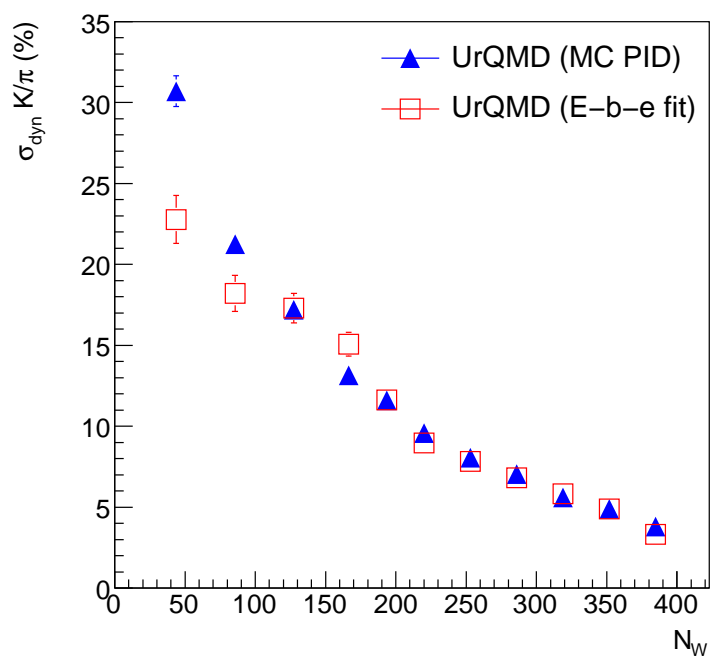

Figure 4: Dynamical fluctuations of the $\mathrm{K} / \pi$ ratio as a function of the number of wounded nucleons in $\mathrm{Pb}+$ $\mathrm{Pb}$ collisions at $158 A \mathrm{GeV}$ beam energy, simulated with UrQMD, for two cases: Monte Carlo based counting (triangles) and event-by-event fit based on simulated $d E / d x$ response (squares).

For both identification procedures the dynamical fluctuations increase with decreasing number of wounded nucleons. But the results for the event-by-event fit start to deviate from the MC reference for less than 200 wounded nucleons, which approximately corresponds to a centrality of $35 \%$. The contribution of the spike at zero in this case exceeds $2 \%$ of all measured events. Thus NA49 data will be analyzed in the centrality range from $0 \%$ to $35 \%$ most central $\mathrm{Pb}+\mathrm{Pb}$ collisions, where the identification procedure does not introduce a bias to the results. 


\subsection{Centrality Dependence}

The measured centrality dependences of the dynamical fluctuations of the $\mathrm{K} / \pi$ and $\mathrm{p} / \pi$ ratios in $\mathrm{Pb}+\mathrm{Pb}$ collisions at $158 \mathrm{~A} \mathrm{GeV}$ beam energy are presented in figure 5.
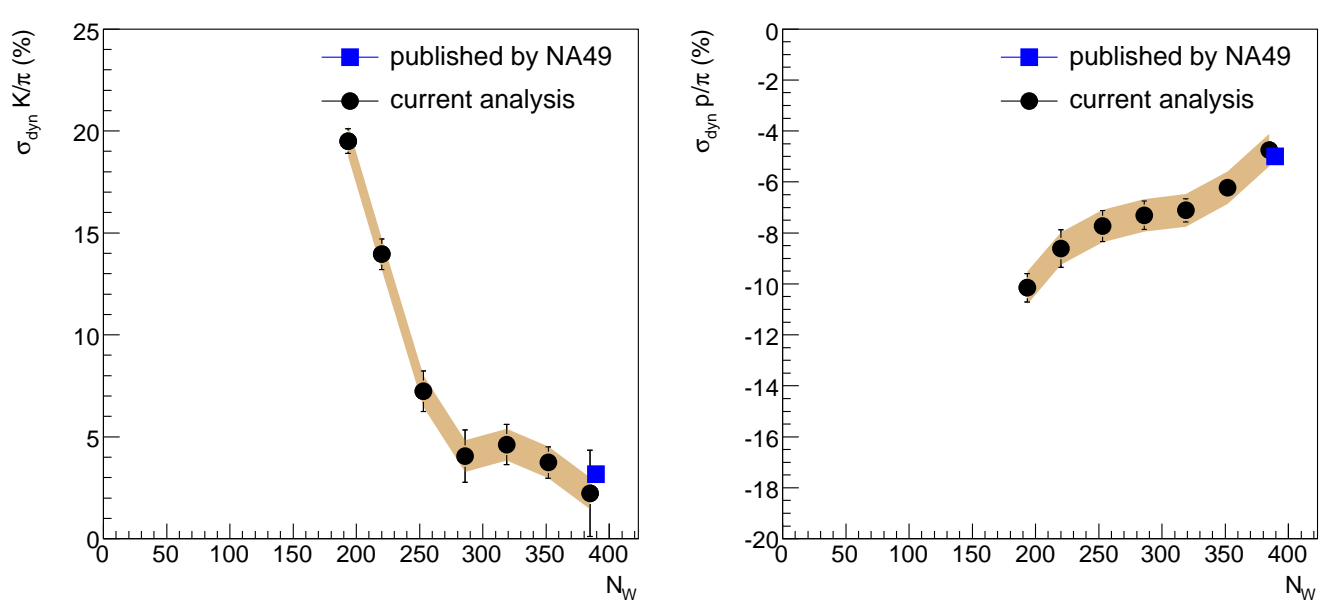

Figure 5: Dynamical fluctuations of the $\mathrm{K} / \pi$ (left picture) and the $\mathrm{p} / \pi$ (right picture) ratios as a function of the number of wounded nucleons in $\mathrm{Pb}+\mathrm{Pb}$ collisions at $158 \mathrm{~A} \mathrm{GeV}$ beam energy. The shaded band shows systematic errors determined from a variation of track cuts.

Figure 6 shows the dynamical fluctuations of the K/p ratio as a function of the number of wounded nucleons in $\mathrm{Pb}+\mathrm{Pb}$ collisions at $158 \mathrm{~A} \mathrm{GeV}$ beam energy. The fluctuations are calculated as mean value from analysis with different track cuts. Their difference is used to estimate the systematic error which is given by the shaded band.

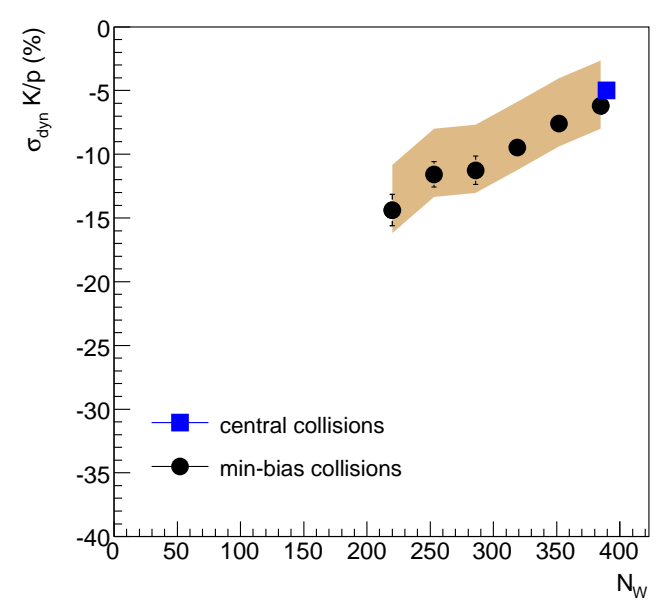

Figure 6: Dynamical fluctuations of the $\mathrm{K} / \mathrm{p}$ ratio as a function of number of wounded nucleons in $\mathrm{Pb}+\mathrm{Pb}$ collisions at $158 \mathrm{~A} \mathrm{GeV}$ beam energy. The shaded band shows systematic errors determined from a variation of track cuts.

The general trend is the same for all considered particle yield ratio fluctuations: the absolute value of the dynamical fluctuations increases towards lower centrality of $\mathrm{Pb}+\mathrm{Pb}$ collisions. 


\subsection{Scaling of Dynamical Fluctuations}

From the definition of the dynamical fluctuations (equation 2.1) one can derive an exact analytical expression, which in case of $\mathrm{K} / \pi$ consists of kaon, pion and correlation terms:

$$
\sigma_{d y n}=\sqrt{\frac{\operatorname{var}\left(N_{K}\right)}{\left\langle N_{K}\right\rangle^{2}}+\frac{\operatorname{var}\left(N_{\pi}\right)}{\left\langle N_{\pi}\right\rangle^{2}}-2 \frac{\operatorname{cov}\left(N_{K}, N_{\pi}\right)}{\left.<N_{K}><N_{\pi}\right\rangle}},
$$

In case of $\mathrm{p} / \pi, N_{K}$ should be substituted with $N_{p}$. As has been argued in $[6,13]$, dynamical fluctuations of the $\mathrm{p} / \pi$ ratio originate dominantly from $\Delta$ resonance decays, which produces correlated protons and pions. The analytical expression for the dynamical fluctuations in this case can be approximated by the correlation term only:

$$
\sigma_{d y n}^{p / \pi} \approx-\sqrt{\frac{\operatorname{cov}\left(N_{p}, N_{\pi}\right)}{<N_{p}><N_{\pi}>}}=-\sqrt{\frac{\left(<N_{p}><N_{\pi}>\right)^{\alpha}}{<N_{p}><N_{\pi}>}},
$$

where we have parametrised the covariance by the product of average multiplicities of protons and pions to the power of $\alpha$. With a strong feeddown from resonances this parameter $\alpha$ is expected to become 0.5 . In case of the $\mathrm{K} / \pi$ ratio we have $N_{K} \ll N_{\pi}$ and the analytical expression for $\sigma_{d y n}$ can be apporoximated by:

$$
\sigma_{d y n}^{K / \pi} \approx \sqrt{\frac{\operatorname{var}\left(N_{K}\right)}{<N_{K}>^{2}}}
$$
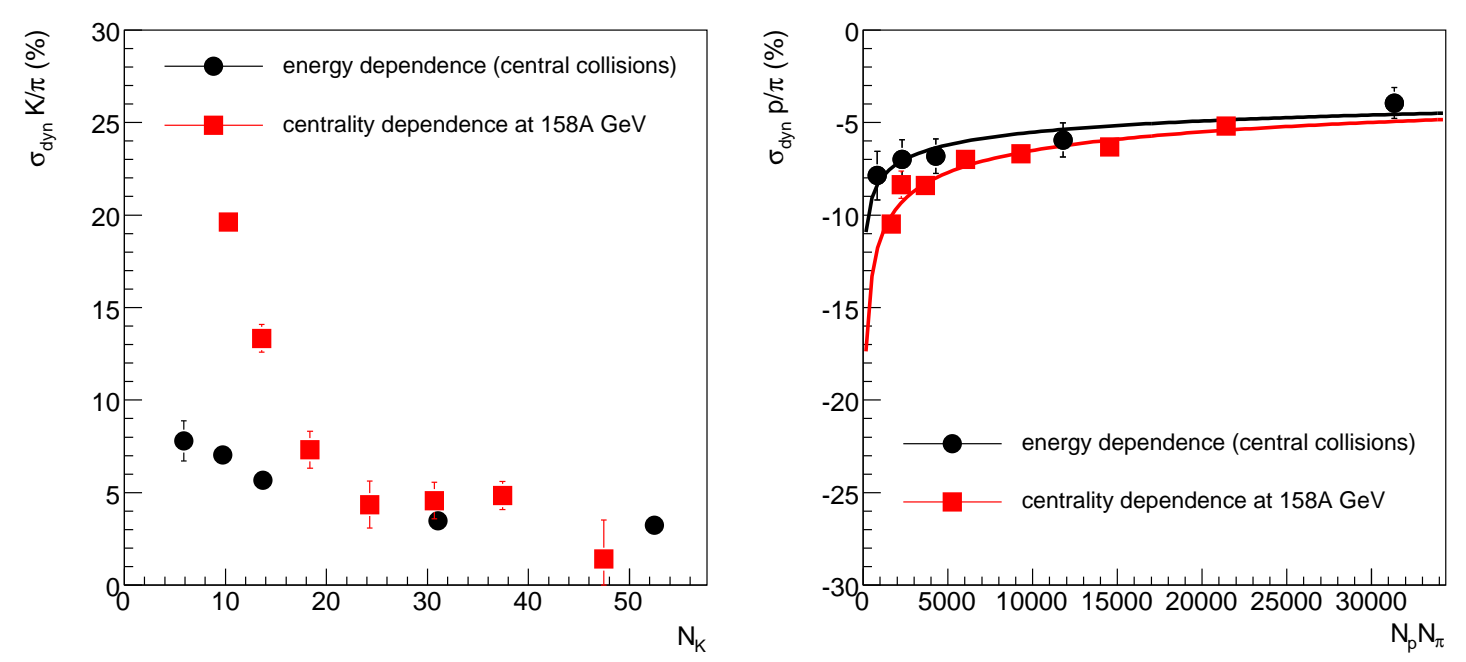

Figure 7: Left picture: dynamical fluctuations of the $\mathrm{K} / \pi$ ratio as a function of $\left\langle N_{K}\right\rangle$ for the energy (circles) and the centrality (squares) dependences. Right picture: dynamical fluctuations of the $\mathrm{p} / \pi$ ratio as a function of $\left\langle N_{p}><N_{\pi}>\right.$ for the energy (circles) and the centrality (squares) dependences. Solid curves show fits according to equation 2.3.

Figure 7 shows the dynamical fluctuations of the $\mathrm{K} / \pi$ ratio as a function of $<N_{K}>$ (left picture) and the dynamical fluctuations of the $\mathrm{p} / \pi$ ratio as a function of $\left\langle N_{p}\right\rangle\left\langle N_{\pi}\right\rangle$ (right picture). 
Data points are taken from the measured energy and centrality dependences for a more loose set of track cuts; the mean number of particles is calculated in the acceptance used to determine the fluctuations. After fitting the dependences of the $\mathrm{p} / \pi$ ratio fluctuations with equation 2.3 , the following $\alpha$ parameters were obtained: $\alpha=0.66 \pm 0.12$ for the energy dependence and $\alpha=0.51 \pm 0.03$ for the centrality dependence. This observation strongly supports the assumption that $\Delta$ resonance decays are the dominant source of dynamical fluctuations of the $\mathrm{p} / \pi$ ratio and give a natural explanation for both, the energy and centrality dependence. As expected also for $\mathrm{K} / \pi$ ratio fluctuations some dependence on $\left\langle N_{K}\right\rangle$ is seen, however the energy and centrality dependence obviously scale differently with $\left\langle N_{K}\right\rangle$. Whether this might be connected to different relative strangeness production in smaller systems [14] requires further studies.

\section{Particle Ratio Fluctuations in CBM}

CBM is the future heavy-ion fixed target experiment at FAIR [15]. Its goal is to measure hadrons and leptons in A + A collisions at beam energies from 10 - $45 \mathrm{AGeV}$. The proposed CBM detector (electron option) is shown in figure 8.

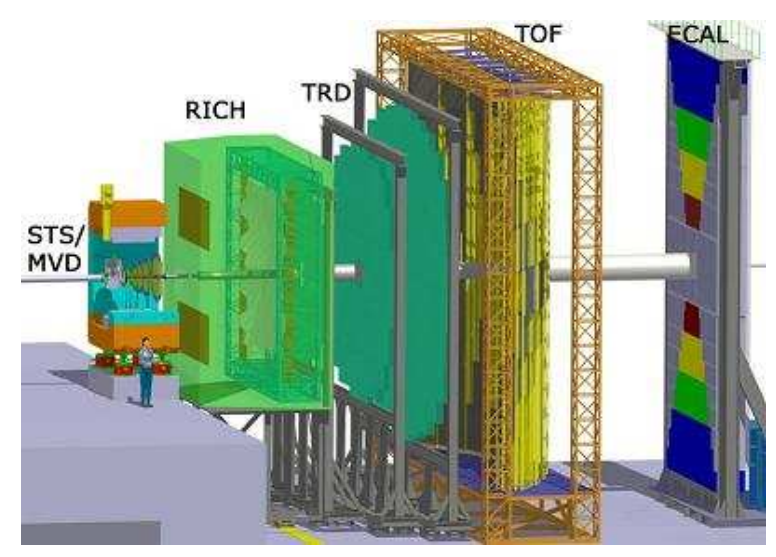

Figure 8: Sketch of the CBM detector.

In this section we will focus on feasibility studies of the measurement of event-by-event fluctuations of the $\mathrm{K} / \pi$ ratio with CBM. In CBM hadrons will be identified using a TOF wall. With a time resolution of better than 80 ps it will be possible to identify particles on the track-by-track level, which together with a large acceptance (larger as compared to the NA49 experiment) should avoid the development of a spike at zero in the eventwise $\mathrm{K} / \pi$ ratio distributions. The distribution of the squared mass versus momentum of reconstructed primary hadrons from UrQMD [12] simulations is shown in figure 9 .

A clean identification of kaons with a pion contamination on the level of $15 \%$ only (integrated) is possible up to momenta of approximately $3 \mathrm{GeV} / \mathrm{c}$.

A certain level of the purity of kaon identification is achieved by using an upper momentum cut. As this of course restricts the phase space acceptance, the effect of this cut on the dynamical fluctuations of the $\mathrm{K} / \pi$ ratio was investigated. As a reference, the results with Monte Carlo (MC) identification but the same upper momentum cut were calculated. The observed dependence of the dynamical fluctuations on the purity of kaon identification i.e. the applied momentum cut is 

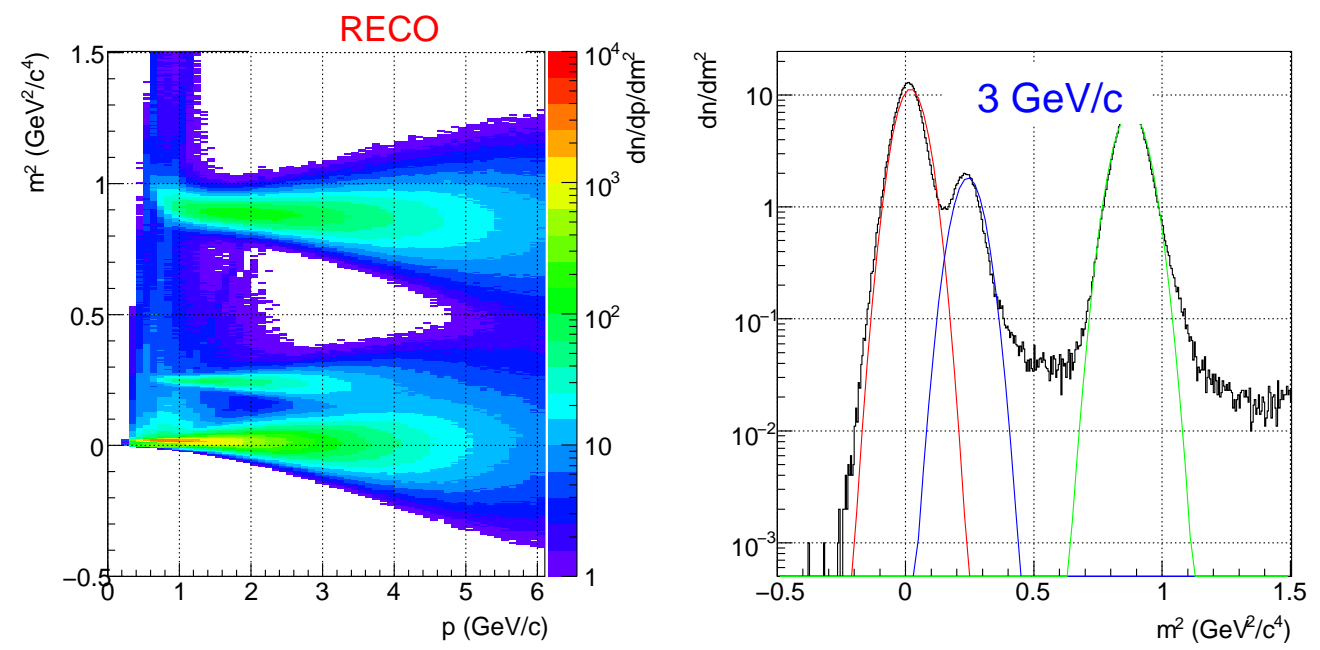

Figure 9: Distribution of the squared mass versus momentum of reconstructed primary hadrons from central $\mathrm{Au}+\mathrm{Au}$ collisions at $25 \mathrm{~A} \mathrm{GeV}$ beam energy (UrQMD simulation). Right picture shows a projection for the momentum of $3 \mathrm{GeV} / \mathrm{c}$. The tails originate from secondary particles and ghost tracks (mismatches).

presented in figure 10 for MC identification and fully reconstructed and identified tracks in the CBM simulation.

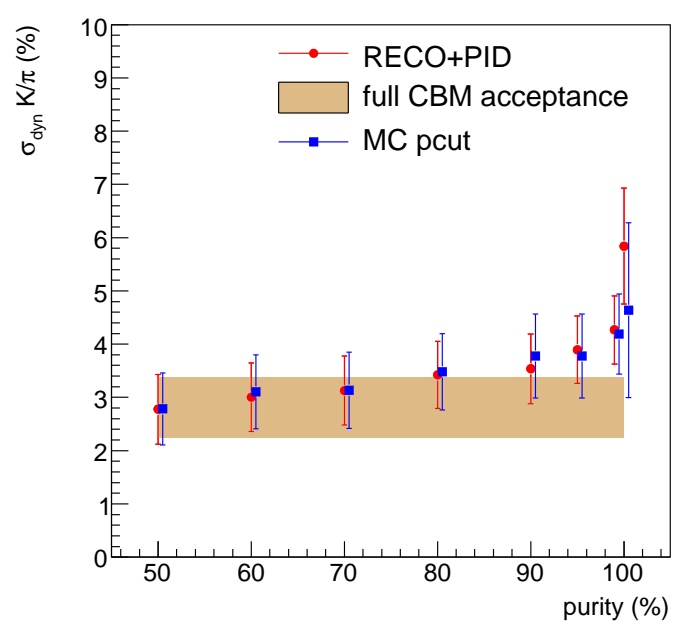

Figure 10: Dynamical fluctuations of the $\mathrm{K} / \pi$ ratio as a function of purity of the kaon identification for the standard identification procedure (circles), Monte Carlo based counting with the upper momentum cut, which corresponds to the chosen purity, (squares) and full CBM acceptance (filled region).

For example the purity requirement of at least $50 \%$ per momentum bin corresponds to a momentum cut of $6 \mathrm{GeV} / \mathrm{c}, 99 \%$ to $3.25 \mathrm{GeV} / \mathrm{c}$. Values of the dynamical fluctuations increase if the momentum cut is more restrictive. Good agreement between results with real identification and PID on the MC level with corresponding momentum cut is observed. The results are closer to the values for the full CBM acceptance in case of lower purity, which corresponds to a more relaxed total momentum cut. 


\section{Summary and Outlook}

The centrality dependences of $\mathrm{K} / \pi, \mathrm{p} / \pi$ and $\mathrm{K} / \mathrm{p}$ ratio fluctuations show increasing absolute values towards lower centrality. A multiplicity dependence is indeed expected and the dependence of the $\mathrm{p} / \pi$ ratio fluctuations on energy and centrality can both together be well described assuming a dominant contribution from $\Delta$ resonance decays. In case of the $\mathrm{K} / \pi$ ratio fluctuations however, the values of the dynamical fluctuations of the $\mathrm{K} / \pi$ ratio for semi-peripheral $\mathrm{Pb}+\mathrm{Pb}$ collisions at $158 \mathrm{~A}$ $\mathrm{GeV}$ are higher than those for central $\mathrm{Pb}+\mathrm{Pb}$ collisions at lower SPS energies at the same values of $\left\langle N_{K}\right\rangle$. In other words: the energy and centrality dependences of the $\mathrm{K} / \pi$ ratio fluctuations scale differently with $\left\langle N_{K}>\right.$.

As dynamical fluctuations in particle emmision are expected to be an important observable for the critical endpoint and current data are not conclusive yet, the future CBM experiment at FAIR prepares to also provide precise evaluation/observation of these fluctuations. Feasibility studies for the measurement of event-by-event fluctuations of the $\mathrm{K} / \pi$ ratio in central $\mathrm{Au}+\mathrm{Au}$ collisions were presented and demonststrate that some of the experimental difficulties in the NA49 analysis can be overcome. The study shows that such a measurement is feasible and that no strong bias from the identification procedure with the TOF wall of CBM is expected.

\section{References}

[1] Z. Fodor and S. D. Katz, JHEP 0404 (2004) 050 [arXiv:hep-lat/0402006].

[2] S. Aoki et al. [JLQCD Collaboration], Phys. Rev. D 72 (2005) 054510 [arXiv:hep-lat/0409016].

[3] S. Ejiri, Phys. Rev. D 77 (2008) 014508 [arXiv:0706.3549 [hep-lat]].

[4] C. R. Allton, S. Ejiri, S. J. Hands, O. Kaczmarek, F. Karsch, E. Laermann and C. Schmidt, Phys. Rev. D 68 (2003) 014507 [arXiv:hep-lat/0305007].

[5] V. Koch, arXiv:0810.2520 [nucl-th].

[6] C. Alt et al. [NA49 Collaboration], arXiv:0808.1237 [nucl-ex].

[7] B. I. Abelev et al. [STAR Collaboration], arXiv:0901.1795 [nucl-ex].

[8] S. Afanasev et al. [NA49 Collaboration], Nucl. Instrum. Meth. A 430 (1999) 210.

[9] S. Wenig [NA49 Collaboration], Nucl. Instrum. Meth. A 409 (1998) 100.

[10] C. Roland, Ph.D. thesis, J. W. G. Universität, Frankfurt am Main, (1999).

[11] D. Kresan, Ph.D. thesis, J. W. G. Universität, Frankfurt am Main, to be submitted in 2009.

[12] S. A. Bass et al., Prog. Part. Nucl. Phys. 41 (1998) 255 [Prog. Part. Nucl. Phys. 41 (1998) 225] [arXiv:nucl-th/9803035].

[13] D. Kresan and V. Friese, PoS C FRNC2006 (2006) 017.

[14] C. Hohne, F. Puhlhofer and R. Stock, Phys. Lett. B 640 (2006) 96 [arXiv:hep-ph/0507276].

[15] CBM Collaboration, http://www.gsi.de/onTEAM/dokumente/public/DOC-2005-Feb-447.html. 